\title{
Relación entre parámetros antropométricos, agarre máximo y velocidad delanzamiento en jugadores jóvenes de waterpolo \\ Relationship among anthropometric parameters, maximal grip and throwing velocity in youth water polo players \\ *Pablo José Borges Hernández, **Encarnación Ruiz Lara, *Francisco Manuel Argudo Iturriaga \\ *Universidad Autónoma de Madrid (España), ** Universidad Católica San Antonio Murcia (España)
}

Resumen. El presente estudio pretende describir un perfil antropométrico en jóvenes waterpolistas masculinos de alto rendimiento deportivo e identificar relaciones significativas entre estas medidas y la fuerza de agarre máxima y la velocidad de lanzamiento. Veinticinco jugadores de waterpolo, con una edad media de $14.8 \pm 1.96$ años, fueron testados, realizándoles una evaluación antropométrica y evaluando la velocidad de lanzamiento en tres ubicaciones espaciales diferentes (centro, derecha e izquierda) desde la línea de 5 metros con oposición del portero y sin ella. También se midió la fuerza de agarre máxima. Se midieron diversas variables antropométricas (masa, altura, envergadura, pliegues cutáneos, diámetros óseos y perímetros musculares). Se procedió con coeficiente de correlación por rangos de Spearman. Y se encontró relación entre el agarre máximo y la velocidad de lanzamiento $(\mathrm{p}=.83 ; \mathrm{p}<.01)$, entre el diámetro biacromial y el agarre máximo $(\mathrm{p}=.53 ; \mathrm{p}<.01)$. Y entre el diámetro biacromial y la velocidad de lanzamiento $(\mathrm{p}=.67 ; \mathrm{p}<.01)$. En conclusión, se considera que los parámetros antropométricos, salvo los pliegues cutáneos tienen relación con la velocidad de lanzamiento y la fuerza máxima de agarre en jóvenes waterpolistas. Asimismo se aprecia una relación considerable entre la velocidad de lanzamiento en la situación con portero y sin portero $(\mathrm{p}=.89$; $\mathrm{p}<.01)$, aunque aún es necesaria mayor investigación.

Palabras clave: waterpolo, cineantropometría, lanzamiento, fuerza de agarre y juventud.

Abstract. This study attempts to explain in a sample of young water polo players an anthropometric profile and identify significant relationships between these measures and the maximum handgrip strength and throwing velocity. Twenty five male water polo players, with a mean age of $14.8 \pm$ 1.96 years, were tested, to perform an anthropometric assessment and evaluating the throwing velocity in three different spaces situations (center, right and left) from the 5 meters line with goalkeeper opposition and without it. Maximum grip was also measured. Various anthropometric variables (weight, height, size, skinfolds, bone and muscle diameter, etc.) were measured. We proceeded with Spearman rank correlation coefficient. And relationship between maximum grip and throwing velocity was found $(\mathrm{p}=.83$; $\mathrm{p}<.01)$, among biacromial diameter and maximum grip ( $\mathrm{p}=.53$; $\mathrm{p}<.01)$ and biacromial diameter and shoot velocity $(\mathrm{p}=.67 ; \mathrm{p}<.01)$. In conclusion, it is considered that the anthropometric parameters, except skinfolds are related to the shoot speed and maximum grip strength in young water polo players. A significant relationship between the throwing velocity in the situation without goalkeeper and with goalkeeper $(\mathrm{p}=.89 ; \mathrm{p}<.01)$ also seen, although further research is still needed.

Key words: water polo, kinanthropometry, throw, hand grip, youth.

\section{Introducción}

El waterpolo es una modalidad deportiva acuática que tiene un alto nivel de exigencia física y psíquica. Requiere el desarrollo de habilidades motrices básicas como saltos, giros y lanzamientos, de ahí que resulte necesario poseer altos niveles de fuerza para desplazarse, golpear, bloquear, empujar y/o mantenerse a flote durante el juego (Smith, 1998; Vila, Ferragut, Argudo, Abraldes, Rodríguez \&Alacid, 2009). Además se hace imprescindible potenciar el pensamiento táctico y la toma de decisiones para formar jugadores de alto nivel deportivo.

Debe señalarse que las variables físicas relacionadas con la valoración antropométrica, composición corporal y somatotipo, desempeñan un papel importante en el desarrollo de un alto rendimiento deportivo. Pero actualmente son escasas las investigaciones en relación a este objeto de conocimiento (García,Alcaraz, Ferragut, Manchado,Abraldes, Rodríguez \& Vila, 2011).

En este sentido, se han encontrado diferentes estudios que han descrito un perfil antropométrico ideal para muchos deportes, pero en waterpolo sólo se conocen unos pocos estudios con tal fin, siendo el realizado con deportistas de la selección española absoluta (Ferragut, Vila, Abraldes, Argudo, Rodriguez \& Alcaraz, 2011) uno de los pocos ejemplos. Por este motivo, este estudio realizado con jugadores jóvenes, intenta determinar un perfil antropométrico que se relacione con una mayor fuerza de agarre y velocidad de lanzamiento; y por tanto un mayor rendimiento deportivo y/o mejor ejecución (Ferragut, Abraldes, Manchado \& Vila, 2015; García, et al., 2011; Gómez, Sabido, GómezValadés, \& Barbado, 2011; Vila, Abraldes \& Rodríguez, 2009). Puesto que Norton y Olds (2001) y Padilla (2010), sugieren que cada deporte e incluso cada posición de juego tienen unas demandas diferentes y únicas, y por tanto unos atributos físicos y fisiológicos propios que determinarán el éxito. De ahí que radique la importancia de este tipo de pruebas para la medida del rendimiento y la detección del talento en

Fecha recepción: 31-05-16. Fecha de aceptación: 27-10-16

Francisco Manuel Argudo

quico.argudo@uam.es edades tempranas.

Por otro lado, las diferentes modificaciones reglamentarias introducidas por la Federación Internacional de Natación Amateur (FINA) en las últimas dos décadas (duración de los periodos de juego, distancias de lanzamientos, tiempo de posesión del balón, etc.). Han dado a lugar a que las características antropométricas de los waterpolistas de élite hayan cambiado (Lloret, 1994, 1995). Sin embargo, únicamente se tiene conocimiento del estudio realizado por Lozovina, Lozovina y Pavièiæ (2012) comparando el perfil antropométrico de jugadores de élite croatas entre 1980 y 2008. En ningún caso se conoce ningún estudio realizado con jugadores en etapas de formación.

Aún así, es de reseñar que estos escasos estudios han permitido detectar la producción de ciertos cambios, tanto a nivel físico como fisiológico, observando una modificación en cuanto al perfil antropométrico de los deportistas durante los últimos años. Pero es necesario contemplar la realización de nuevos estudios que ratifiquen estos datos, así como comparar los resultados con la evolución en este mismo aspecto en otros deportes. En la actualidad, los waterpolistas poseen una mayor altura y extremidades más largas, una cintura más delgada y los hombros más anchos (Lozovina, et al., 2012). Sin embargo, la masa corporal ha permanecido sin cambios, mientras se ha incrementado la masa muscular de los atletas.

Otro de los aspectos claves que se puede observar en estos últimos años, debido a la variación antropométrica de los jugadores de élite, es la variación entre el tipo de lanzamiento efectuado y la velocidad de los mismos, primando los lanzamientos tensos y a máxima velocidad (Argudo, 2002; García, 2009). Por lo que recientemente, entre las modificaciones propuestas por la FINA, varias federaciones nacionales han solicitado la modificación del tamaño y peso del balón; para otorgar, si cabe aun mayor velocidad a los lanzamientos, y por tanto mejorar el espectáculo con el aumento de los goles anotados (Argudo, 2002; Argudo \& García, 2013). Puesto que una de las máximas en la consecución del éxito de este deporte radica en la puntería y velocidad de los lanzamientos a portería (Van Muijen, Joris, Kemper, \& Van Ingen Schenau, 1991), ya que el $90 \%$ de los goles se obtienen con lanzamientos de este tipo (Bloomfield. Blanksby, Ackland \& Allison, 1990). 
En este sentido, tradicionalmente, se ha estimado que la velocidad de lanzamiento depende de tres factores (Joris, van Muijen, van Ingen Schenau \& Kemper, 1985; Van Muijen, et al., 1991; Rivilla, Sampedro, Navarro \& Gómez, 2010), la técnica del lanzamiento, la coordinación temporal de las acciones de los diferentes segmentos corporales y la fuerza y potencia de los músculos del tren superior e inferior. Igualmente, se considera que el grado de precisión podría ser un factor de influencia en la velocidad de lanzamiento.

Pero lamentablemente, tal y como afirma Rivilla et al. (2010), existen escasas investigaciones que analicen la relación y diferencias entre velocidad de lanzamiento con oposición y sin ella para corroborarlo. Debido a la propia idiosincrasia de este deporte que se desarrolla en un medio para el cual el ser humano no está preparado, lo que dificulta en gran medida la evaluación de las capacidades fisiológicas de sus practicantes (Hohmann \& Frase, 1992). Sin embargo, si se establecieran una serie de test en los que se diera cabida a pruebas antropométricas y fisiológicas, se podría llegar a determinar un perfil integral del jugador de waterpolo de élite (Tsekouras, Kavouras, Campagna, Kotsis, Syntosi, Papazoglou \& Sidossis, 2005).

En este sentido, cobra importancia el estudio de la técnica y la táctica en este tipo de deportes (Argudo, Ruiz \& Abraldes, 2010) en los que se ha demostrado (Gorostiaga, Granados, Ibañez, Gonzalez-Badillo \& Izquierdo 2006; Gorostiaga, Granados, Izquierdo, Ibañez \& Bonnabau 2007; Hohmann \& Frase, 1992; Wallace \& Cardinale, 1997) la relevancia del control del balón, vinculado a la capacidad de agarre del mismo, y la velocidad y precisión de lanzamiento, la cual se verá condicionada por el movimiento del brazo y la velocidad de liberación del balón, donde el agarre tiene una importancia elevada y ello repercute en el éxito deportivo alcanzado.

Así pues, el objetivo de este estudio es doble: 1) definir un perfil antropométrico de jóvenes waterpolistas y 2) identificar si existen relaciones significativas entre las características antropométricas, la velocidad de lanzamiento y la fuerza de agarre máxima.

\section{Metodología}

\section{Participantes}

Para la realización de este estudio participaron 25 deportistas que competían en la máxima categoría a nivel nacional $\left(M_{\text {edad }}=14.80\right.$; $D T=1.96)$, siendo los 25 participantes, hombres y con lateralidad diestra, de los cuales 9 eran infantiles $\left(M_{\text {masa }}=49.9 ; D T=7.99\right.$ y $\left.M_{\text {altura }}=159.16 ; D T=10.74\right), 11$ cadetes $\left(M_{\text {masa }}^{\text {masa }}=59.56 ; D T=8.09 \mathrm{y}\right.$ $\left.M_{\text {altura }}^{\text {altura }}=171.48 ; D T=5.99\right)$ y 5 juveniles $\left(M_{\text {masa }}^{\text {masa }}=63.12 ; D T=5.66 \mathrm{y}\right.$ $\left.M_{\text {altura }}=170.92 ; D T=6.33\right)$. La muestra fue seleccionada mediante muestreo incidental por el único motivo de acceso viable. La participación fue voluntaria y consentida; y se respetó la Declaración de Helsinki en todos sus términos.

\section{Instrumentos ymedidas}

Se utilizó el protocolo Internacional de la Sociedad de Cineantropometría (ISAK). Aplicado por técnicos formados en dicha metodología, para determinar cada uno de los perfiles antropométricos de los deportistas. Los jugadores fueron medidos durante una única sesión con vestimenta ligera, sin calzado y sobre el lado derecho del cuerpo, a primera hora de la mañana, tras un desayuno ligero en los momentos previos a la realización de una sesión de entrenamiento.

El orden seguido para registrar las características físicas fue: altura, masa corporal, envergadura, pliegues cutáneos, perímetros corporales y diámetros esqueléticos, superando en poco más de 30 las mediciones en el programa antropométrico. Los instrumentos utilizados fueron:

- Plataforma nivelada (Seca, Barcelona, España) con una precisión de $.01 \mathrm{~kg}$ y $.001 \mathrm{~m}$, para el masa y la altura, respectivamente.

- Goniómetro Holtain Skinfold Calliper (Holtain, Crymych, United Kingdom) con una precisión de $10 \mathrm{~g} / \mathrm{mm}^{-2}$ de presión constante para los ocho pliegues cutáneos (tríceps, subescapular, bíceps, axilar, abdominal, cresta ilíaca, suprailíaco, cara anterior del muslo y de la pierna).
- Cinta métrica (Lufkin Executive Thinline, Lufkin, TX, USA) para las medidas de seis perímetros corporales (brazo relajado, brazo flexionado y tenso, mesoesternal, cintura, cadera, pierna), a los que se añade la medida de la longitud de la mano y el pie.

- Antropómetro (GPM, Suiza) para la medida de siete diámetros esqueléticos (biacromial, bileocrestal, mesoesternal, pecho, biecondílohumeral, bicondíleofemoral y biestiloideo de la muñeca).

Todas las medidas se realizaron en dos ocasiones (tres para los pliegues cutáneos) por personal acreditado ISAK, considerando un error de medida técnica menor al $2 \%$ para los pliegues cutáneos y menor al 1\% para los diámetros óseos y perímetros corporales.

Como consecuencia de las variables medidas se obtuvieron otros datos:

- El índice de masa corporal (IMC) resultado de dividir la masa (kg) por la altura $\left(\mathrm{m}^{2}\right)$.

- El porcentaje de grasa corporal, estimado partir de las mediciones de los pliegues cutáneos usando el método descrito por Lee, Wang, Heo, Ross, Janssen y Heymsfield (2000).

- La densidad corporal, medida a través de la fórmula de Wither, Craig, Bourdon y Norton (1987). Densidad $=(1,0988-$ 0,0004*(X1), donde X1=»7 pliegues cutáneos (tríceps, subescapular, bíceps, supraespinal, abdominal, muslo y pierna).

- La masa libre de grasa (kg) según el método descrito por Martin, Spenst, Drinkwater y Clarys (1990).

- El somatotipo a partir de las medidas antropométricas, siguiendo el método definido por Carter y Heath (1990).

A continuación, y para el estudio de la fuerza isométrica de agarre se les permitió a los jugadores que realizaran tres pruebas con el aparato de medida, un dinamómetro manual (TKK 5401, Japón), con una sensibilidad de 1 kilogramos de fuerza (Kgf), para que se familiarizaran. A continuación de éstas, y tras un descanso de tres minutos, con el fin de minimizar el efecto de la fatiga, se realizaron dos medidas de la fuerza máxima de agarre con 1 minuto de descanso entre cada medida. Para ello se solicitó a los deportistas, que en posición de pie, con el aparato en paralelo al cuerpo, utilizando su brazo dominante y sin movimiento del brazo o de la muñeca ejercieran la máxima fuerza posible. Se utilizó el promedio para los análisis posteriores.

Finalmente, y con el objeto de medir la velocidad de lanzamiento, se registraron seis lanzamientos (dos para cada condición), utilizando cada jugador su propia técnica, desde la línea de 5 metros en tres condiciones diferentes: 1) zona derecha, 2) zona central; 3) zona izquierda, con oposición (portero) o sin oposición. Para esta prueba se utilizó el balón estándar oficial y reglamentario para cada categoría: Talla 4 para infantiles (Masa $=.4 \mathrm{~kg}$, Diámetro $=.67 \mathrm{~m}$ y Presión $=90 \mathrm{kPa}$ ) y Talla 5 para cadetes y juveniles (Masa $=.45 \mathrm{~kg}$, Diámetro $=.71 \mathrm{~m}$ y Presión=97kPa), tras veinte minutos de calentamiento. Para ello, se colocó detrás de la portería y de forma perpendicular a la dirección de los jugadores una pistola radar (Stalker-Pro Inc., Plano), con $100 \mathrm{~Hz}$ de frecuencia récord y una sensibilidad de $.045 \mathrm{~m} / \mathrm{s}$.

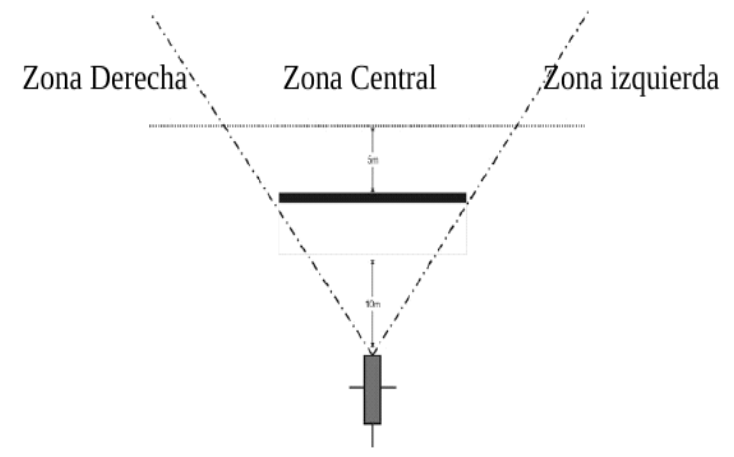

anzamientos 


\section{Procedimiento}

La investigación ha seguido un diseño descriptivo y correlacional de carácter transversal, constituyéndose como un Estudio Instrumental siguiendo un diseño ex post facto retrospectivo (Montero \& León, 2007).

Se contactó con la dirección deportiva del club. Todos los tutores o responsables legales de los deportistas que aceptaron formar parte del estudio firmaron el correspondiente consentimiento informado. Los deportistas fueron avisados de que les serían medidos diferentes segmentos corporales, así como que se estudiaría la velocidad de lanzamiento y la fuerza isométrica de agarre manual. Siendo el objetivo del estudio obtener el perfil antropométrico del deportista y conocer la influencia de este perfil y la fuerza de agarre, en la efectividad y velocidad de los lanzamientos.

El protocolo utilizado para este estudio consistió en realizar las medidas corporales, siguiendo el orden de presentación desarrollado anteriormente. A continuación, los deportistas disfrutaron de 20 minutos de calentamiento en el agua y una explicación acerca de las diferentes condiciones de lanzamientos consideradas, así como tiempo suficiente para desarrollar algún lanzamiento de prueba. A modo de resumen, y para la sección de la velocidad de lanzamiento, el protocolo seguido fue el integrado por dos series de dos lanzamientos cada una, con 25-30 segundos de descanso entre cada uno (Gómez, et al., 2011).Así como se dejaron entre 2 minutos y medio y 3 minutos entre cada par de registros para evitar la fatiga. Con objeto de estimular y que no decayera el estado de ánimo entre los deportistas, se les iba informando de la velocidad alcanzada tras cada lanzamiento.

\section{Análisis estadístico de los datos}

Con la intención de describir el perfil cineantropométrico se estimó de cada variable su mediana (Med) y el $1^{\circ}$ y $3^{\circ}$ cuartil. Se analizó la normalidad de las distribuciones mediante la prueba de Shapiro-Wilk. Para analizar el grado de covariación entre las medidas antropométricas. la velocidad de lanzamiento y la fuerza máxima de agarre, se utilizó el coeficiente de correlación de Spearman (p) puesto que los datos son no paramétricos. Este estadístico se interpretó de acuerdo con Salkind (1999), considerando correlaciones muy bajas entre .00 y .20; bajas entre .21 y .40; moderadas entre .41 y .60; altas entre .61 y .80 y muy altas entre .81 y 1 . Los análisis fueron realizados con el paquete estadístico R(1.36.15), estableciendo un nivel de confianza del $95 \%(p<.05)$.

\section{Resultados}

Con respecto a los análisis descriptivos, la tabla 1 , muestra los valores encontrados en este estudio: Mediana (Med) y el $1^{\circ}$ y $3^{\circ}$ cuartil, de las mediciones realizadas con la intención de medir las características antropométricas de los jóvenes waterpolistas. En esta tabla se pueden apreciar diferencias, si se comparan con resultados previos en jugadores de élite de waterpolo español (Ferragut, Vila, Abraldes, Argudo, Rodriguez \& Alcaraz, 2011) considerando las variables: edad, altura, masa, pliegues corporales, diámetros y perímetros musculares.

La tabla 2, muestra los datos descriptivos obtenidos en relación a la fuerza de agarre máxima de los deportistas (Din) y la velocidad de lanzamiento (Vel). Los resultados indican que a mayor edad se obtiene mayor índice de fuerza $\left(\mathrm{Din}_{\text {infantil }}=23.97 \mathrm{Kgf}\right.$; Din cadete $=29.02 \mathrm{Kgf}$; Din $_{\text {juvenil }}=32.86 \mathrm{Kgf}$ ), así como mayor velocidad de lanzamiento (Vel ${ }_{\text {infan }}$ $=13.68 \mathrm{~m} / \mathrm{s} ; \mathrm{Vel}_{\text {cadete }}=16.92 \mathrm{~m} / \mathrm{s} ; \mathrm{Vel}_{\text {juvenil }}=17.50 \mathrm{~m} / \mathrm{s}$ ).

Tal y como se detalla en la tabla 3 , se observa una relación significativa, elevada y positiva entre la velocidad de lanzamiento, sin oposición $(\mathrm{p}=.66 ; p<.01)$ y con oposición ( $\mathrm{p}=.73 ; p<.01)$ y la envergadura. También se aprecia relación ( $\mathrm{p}=.56 ; p<.01)$ entre la talla y los lanzamientos sin oposición; $y(\mathrm{p}=.68 ; p<.01)$ si se considera la variable oposición. Y un $\mathrm{p}$-valor $(\mathrm{p}=.71 ; p<.01)$ entre la masa y velocidad de lanzamiento $\sin$ oposición y ( $\mathrm{p}=.71 ; p<.01)$ con oposición.

Del mismo modo, estas tres variables arrojan resultados significativos al realizar una correlación, mediante rho de Spearman, entre la fuerza máxima de agarre y las variables antropométricas consideradas. Presentando un valor rho elevado y positivo al comparar la dinamometría

\begin{tabular}{|c|c|c|c|c|c|c|c|c|c|c|c|c|}
\hline \multirow{2}{*}{ Medidas } & \multicolumn{3}{|c|}{ Deportistas $(n=25)$} & \multicolumn{3}{|c|}{ Infantiles $(n=9)$} & \multicolumn{3}{|c|}{ Cadetes $(n=11)$} & \multicolumn{3}{|c|}{ Juveniles $(n=5)$} \\
\hline & Med & 1er Сиа & Зег Сиа & Med & 1er Сua & Зег Сиа & Med & 1er Сиа & 3er Cua & Med & 1er Сua & 3er Сua \\
\hline Edad (años) & 15 & 13 & 16 & 13 & 12 & 13 & 15 & 15 & 16 & 17 & 17 & 18 \\
\hline masa (kg) & 57.3 & 51 & 64.7 & 49.2 & 44.1 & 57.3 & 61.5 & 53.15 & 66.95 & 64.7 & 57.3 & 66.6 \\
\hline Altura $(\mathrm{cm})$ & 167.5 & 162.8 & 173 & 155.2 & 149.3 & 167.5 & 171.5 & 165.8 & 174.7 & 172.2 & 167.1 & 173 \\
\hline Talla Sentado $(\mathrm{cm})$ & 120.2 & 116 & 122.2 & 114.7 & 111.4 & 119.2 & 121 & 117.2 & 123 & 122 & 121.2 & 124 \\
\hline Envergadura (cm) & 173 & 165.5 & 181 & 157 & 153.1 & 172.9 & 174.9 & 171.85 & 179.25 & 183.1 & 173.1 & 186.2 \\
\hline IMC $\left(\mathrm{kg}^{*} \mathrm{~m}-{ }^{2}\right)$ & 20.52 & 18.5 & 21.82 & 20.41 & 18.18 & 20.85 & 20.37 & 18.48 & 22.4 & 21.66 & 21.58 & 21.82 \\
\hline masa libre de grasa $(\mathrm{kg})$ & 9.55 & 7.23 & 10.9 & 9.9 & 7.23 & 11.26 & 7.91 & 5.55 & 10.42 & 9.76 & 7.6 & 10.26 \\
\hline masa libre de grasa (\%) & 16.86 & 10.62 & 19.05 & 19.05 & 16.86 & 22.51 & 11.62 & 9.47 & 18.02 & 14.65 & 13.29 & 16.84 \\
\hline \multicolumn{13}{|c|}{ Pliegues Corporales } \\
\hline Tríceps (mm) & 10.8 & 6.9 & 15.2 & 14 & 12.4 & 15.2 & 7.6 & 6.5 & 13.3 & 8.8 & 6.9 & 10 \\
\hline Subescapular (mm) & 8 & 6.7 & 9.8 & 9.8 & 6.8 & 13 & 7.6 & 6.3 & 8.3 & 9 & 6.9 & 9.2 \\
\hline Bíceps (mm) & 4.9 & 3.7 & 7.9 & 8.6 & 6.6 & 11.4 & 4.3 & 3.55 & 6.7 & 4.6 & 4.4 & 4.6 \\
\hline Cresta Iliaca (mm) & 13 & 7.8 & 18.6 & 18.6 & 8.2 & 19.8 & 10.6 & 7.65 & 16.45 & 13 & 9 & 13.8 \\
\hline Supraespinal (mm) & 8.4 & 6.1 & 12.8 & 11.4 & 6.3 & 14 & 6.8 & 5.4 & 9.75 & 7.6 & 6.1 & 8.4 \\
\hline Abdominal (mm) & 12.8 & 7.2 & 18.4 & 18.4 & 6.8 & 20.6 & 11.2 & 7.2 & 13.8 & 12.8 & 10.9 & 14.6 \\
\hline Muslo (mm) & 15.1 & 9.4 & 18.8 & 21.1 & 17.7 & 21.8 & 11.4 & 8.15 & 17.1 & 12.6 & 12.1 & 12.8 \\
\hline Pierna Medial (mm) & 12 & 8 & 16.2 & 14.8 & 12 & 16.2 & 8 & 7 & 16.3 & 9.2 & 9.1 & 11.8 \\
\hline Densidad (Whiters, 1987) & 1.06 & 1.06 & 1.07 & 1.06 & 1.05 & 1.06 & 1.07 & 1.06 & 1.08 & 1.07 & 1.06 & 1.07 \\
\hline MME (Lee, 2000) & 79.36 & 73.5 & 88.95 & 68.22 & 63.89 & 76 & 85.07 & 78.68 & 90 & 88.95 & 80.4 & 89.06 \\
\hline \multicolumn{13}{|c|}{ Perímetros musculares } \\
\hline Brazo Relajado (cm) & 26.4 & 24.5 & 29 & 24.5 & 23.2 & 25.5 & 28.9 & 25.25 & 29.8 & 29 & 28.4 & 30 \\
\hline Brazo Contraido (cm) & 28.1 & 26.8 & 31.1 & 26.9 & 25.2 & 27.9 & 29 & 27.05 & 31.55 & 31 & 30.9 & 31.2 \\
\hline Mesoesternal (pecho) (cm) & 83 & 76.5 & 91.2 & 76 & 72.7 & 79.7 & 85.6 & 82.25 & 92.8 & 91.2 & 89 & 92.7 \\
\hline Cintura (cm) & 72.3 & 69.4 & 74.5 & 69.4 & 67 & 74 & 72.6 & 69.6 & 75.05 & 74.3 & 70.7 & 74.5 \\
\hline Cadera $(\mathrm{cm})$ & 87.5 & 81.6 & 90.2 & 83 & 80 & 85.3 & 89.2 & 81.7 & 90.5 & 91.7 & 87.5 & 95.5 \\
\hline Pierna (máx.) (cm) & 49.3 & 47.2 & 52.4 & 47.2 & 45.4 & 48.5 & 51.5 & 48.75 & 53.75 & 52.4 & 50.5 & 52.5 \\
\hline Longitud Mano (cm) & 18.4 & 17.2 & 19.4 & 16.9 & 16.3 & 17.7 & 19.2 & 18.2 & 19.75 & 18.6 & 18.4 & 19.8 \\
\hline Longitud Pie $(\mathrm{cm})$ & 25.9 & 24.1 & 26.9 & 24.1 & 23.1 & 25.8 & 26.6 & 25.55 & 27.1 & 25.9 & 24.3 & 27.3 \\
\hline \multicolumn{13}{|c|}{ Diámetros óseos } \\
\hline Diámetro Biacromial (cm) & 37.5 & 35.1 & 39.1 & 34.4 & 32.3 & 36.7 & 38.5 & 37.35 & 39.65 & 39.1 & 38.2 & 40.6 \\
\hline Diámetro Bileocrestal (cm) & 27.3 & 25.9 & 28.7 & 26.2 & 25.7 & 28.7 & 26.9 & 26.25 & 28.2 & 28 & 27.3 & 29.8 \\
\hline Diámetro Mesoesternal (cm) & 29.2 & 28 & 31.2 & 27 & 24.5 & 28.5 & 30.5 & 28.7 & 31.2 & 31.3 & 31.2 & 32.8 \\
\hline Diámetro A-P Pecho (cm) & 18.7 & 17.7 & 20.5 & 17.9 & 16.9 & 18.3 & 19.6 & 18.55 & 20.35 & 21.7 & 20.8 & 21.8 \\
\hline Diámetro Húmero (cm) & 6.7 & 6.6 & 7 & 6.5 & 6.2 & 6.8 & 6.9 & 6.7 & 7.15 & 6.6 & 6.6 & 6.6 \\
\hline Diámetro Fémur (cm) & 9.5 & 9.2 & 9.6 & 9.1 & 9 & 9.6 & 9.5 & 9.45 & 9.55 & 9.5 & 9.2 & 9.5 \\
\hline Diámetro Biestiloideo (cm) & 5.4 & 5.1 & 5.5 & 5.1 & 4.9 & 5.4 & 5.5 & 5.2 & 5.6 & 5.4 & 5.4 & 5.5 \\
\hline \multicolumn{13}{|c|}{ Somatotipo } \\
\hline Endomorfia & 3.66 & 2.01 & 5.27 & 4.66 & 3.57 & 6.34 & 2.29 & 1.85 & 4.01 & 3.06 & 2.54 & 3.92 \\
\hline Mesomorfía & 5.18 & 4.3 & 5.55 & 5.03 & 3.59 & 5.45 & 5.25 & 4.44 & 6.25 & 5.18 & 5.13 & 5.33 \\
\hline Ectomorfia & 3.15 & 2.35 & 3.93 & 2.9 & 2.17 & 3.87 & 3.93 & 2.28 & 4.19 & 2.82 & 2.66 & 3.15 \\
\hline
\end{tabular}


Tabla 2

Valores descriptivos de fuerza de agarre y velocidad de lanzamiento de jugadores jóvenes de waterpolo (Med. 1er y 3er Cuartil). Variables $\quad$ Deportistas $(\mathrm{n}=25) \quad$ Infantiles $(\mathrm{n}=9) \quad$ Cadetes $(\mathrm{n}=11) \quad$ Juveniles $(\mathrm{n}=5)$ \begin{tabular}{ccccccccccccc}
\cline { 2 - 10 } & Med & 1er Cua & 3er Cua & Med & 1er Cua & 3er Cua & Med & 1er Cua & 3er Cua & Med & 1er Cua & 3er Cua \\
\hline Din_Der. (Kgf) & 28.45 & 25.15 & 36 & 24.25 & 21.4 & 27.4 & 29.05 & 27.53 & 34.08 & 36.2 & 28.45 & 37.65
\end{tabular} $\begin{array}{lllllllllllll}\text { Din_Izq. (Kgf) } & 24.85 & 21.1 & 29.5 & 20.35 & 18.7 & 24 & 26.05 & 23.88 & 31.3 & 29.5 & 27.35 & 38.15 \\ \text { Di__l. } & 27.23 & 23.53 & 32.68 & 22.4 & 20.05 & 25.45 & 27.43 & 25.81 & 32.51 & 32.68 & 28.98 & 37.18\end{array}$ $\begin{array}{lllllllllllll}\text { Dinamometría } & 27.23 & 23.53 & 32.68 & 22.4 & 20.05 & 25.45 & 27.43 & 25.81 & 32.51 & 32.68 & 28.98 & 37.18\end{array}$ $\begin{array}{lllllllllllll}\text { Lanz. } 1 \mathrm{ZI}(\mathrm{m} / \mathrm{s}) & 14.72 & 13.33 & 16.67 & 13.06 & 12.22 & 14.44 & 14.72 & 13.47 & 16.39 & 17.22 & 16.67 & 17.78\end{array}$ $\begin{array}{llllllllllllll}\text { Lanz. } 2 \text { ZC (m/s) } & 15.56 & 13.89 & 16.39 & 14.44 & 12.78 & 15.56 & 15.28 & 14.17 & 16.11 & 17.5 & 16.94 & 17.78\end{array}$ $\begin{array}{cccccccccccccc}\text { Lanz. 3 ZD (m/s) } & 14.72 & 14.17 & 16.94 & 13.61 & 12.78 & 14.44 & 14.72 & 14.31 & 15.56 & 17.78 & 17.22 & 17.78 \\ \text { Sin Oposición } & 15.09 & 13.8 & 16.48 & 13.52 & 12.78 & 15.09 & 15 & 13.84 & 15.83 & 17.13 & 17.04 & 17.78\end{array}$ $\begin{array}{llllllllllllll}\text { Lanz. } 4 \text { ZI (m/s) } & 15.28 & 13.33 & 15.83 & 13.61 & 11.94 & 13.89 & 15.28 & 13.19 & 15.97 & 15.83 & 15.56 & 16.67\end{array}$ $\begin{array}{lllllllllllll}\text { Lanz } 5 \mathrm{ZC}(\mathrm{m} / \mathrm{s}) & 15.28 & 14.44 & 16.67 & 15 & 12.78 & 15.28 & 16.39 & 14.72 & 16.67 & 17.22 & 16.94 & 17.22\end{array}$ $\begin{array}{lllllllllllll}\text { Lanz. } 6 \text { ZD (m/s) } & 15.28 & 12.78 & 16.94 & 13.06 & 12.5 & 15 & 16.67 & 13.89 & 17.08 & 16.39 & 15.56 & 18.06\end{array}$ \begin{tabular}{lllllllllllll} 
Oposición & 15.09 & 13.61 & 16.39 & 13.8 & 12.5 & 14.72 & 16.2 & 13.61 & 16.62 & 16.39 & 15.83 & 17.31 \\
\hline
\end{tabular} \begin{tabular}{lllllllllllll} 
Velocidad Lanz. & 15.33 & 13.61 & 16.16 & 13.8 & 12.69 & 14.91 & 15.69 & 13.91 & 16.13 & 17.22 & 16.44 & 17.31 \\
\hline Leyenda: Med: mediana de las puntuaciones. 1er y & 3er Cuar: primer o Tercer Cuartil de las puntuaciones. Lanz: Promedio de la
\end{tabular} velocidad de los lanzamientos efectuados en función de su localización (ZI. zona izquierda. ZC. zona central y ZD. zona derecha). Sin Oposición: promedio de la velocidad de lanzamientos realizados en la condición "sin portero". Oposición: promedio de la velocidad de lanzamientos realizados en la condición "con portero".

con brazo dominante y la envergadura $(\mathrm{p}=.71 ; p<.01)$; dinamometría y talla $(\mathrm{p}=.60 ; p<.01) ; \mathrm{y}(\mathrm{p}=.71 ; p<.01)$ al contrastar la masa y los valores de fuerza máxima.

Por otro lado, también es de destacar la aparición de significación estadística al relacionar el índice de masa corporal (IMC) y la velocidad de lanzamiento [ $\mathrm{p}=.4 ; p<.05]$, pero no se aprecia significación entre el IMC y la fuerza máxima de agarre ( $\mathrm{p}=.37 ; p>.05)$.

Asimismo, se aprecia significación estadística al analizar las relaciones existentes entre diámetro biacromial y velocidad de lanzamiento [p=.64; $p<.01$, (sin oposición) y p=.67; $p<.01$ (con oposición)], entre diámetro biacromial y fuerza máxima de agarre $(\mathrm{p}=.57 ; p<.01)$, entre diámetro bilieocrestal y velocidad de lanzamiento $[\mathrm{p}=.47 ; p<.05$ (sin oposición), $\mathrm{p}=.41 ; p<.05$ (con oposición)], pero no se aprecia significación entre el diámetro biliocrestal y la fuerza máxima de agarre $(\mathrm{p}=.32$; $p>.05)$.

Respecto a los análisis correlación entre fuerza de agarre máxima y velocidad de lanzamiento. Los resultados indican que todas las variables consideradas presentan significación estadística $(p<.01)$, destacando las altas correlaciones halladas, entre velocidad de lanzamiento y dinamometría ( $\mathrm{p}=.79 ; p<.01$ ). Asimismo, destacan las correlaciones de magnitud elevada y positivas entre la velocidad de lanzamiento sin oposición y con oposición $(\mathrm{p}=.89 ; p<.01)$.

\section{Discusión}

Este trabajo se ha centrado en presentar y definir un perfil antropométrico relacionado con el máximo rendimiento de jóvenes waterpolistas, con la intención de identificar la existencia de relación entre las características antropométricas, la velocidad de lanzamiento, y la fuerza de agarre.

Al comparar el perfil antropométrico medido en los deportistas de este estudio, se aprecian diferencias con los hallados en waterpolistas adultos (Ferragut, et al., 2011), así como con los perfiles predominantes en otros deportes, tal y como afirman Norton y Olds (2001) y Padilla (2010).

De este modo, y en relación a la altura, se aprecia en este estudio, que los waterpolistas analizados son más altos que las gimnastas estudiadas por Leyton, Luis, Sabido y Morenas (2012), así como los participantes en el estudio de Domínguez, Sánchez, Rodríguez y González (2015). Por otro lado, presentan una altura similar a las jugadoras de pádel analizadas por Pradas, Cachón, Ortín, Quintas, Arraco y Castellar (2014), pese a tener menor edad. Sin embargo, se observa una altura inferior a socorristas (Abraldes, Rodríguez, Ferragut \& Vila, 2014) y los jugadores adultos de balonmano analizados por Piscitelli, Milanese, Sandri, Cavedon y Zancanaro (2016).

Comparando la masa corporal, los waterpolistas presentan valores inferiores a los reportados por Vila et al. (2009), en jugadores de balonmano en edad juvenil de la región de Murcia, así como los analizados por Piscitelli et al. (2016); y que los modelos publicitarios costaricenses analizados por Chacón-Araya, Fernández-Campos y Moncada-Jiménez (2016).

Considerando la envergadura, esta es inferior a la observada en socorristas (adultos) por Abraldes et al. (2014), y ligeramente inferior a la de los jugadores de balonmano juveniles analizados por Vila et al. (2009). Por último, y teniendo en cuenta los pliegues corporales, se puede observar pliegues de menor tamaño que los hallados por Vila et al. (2009) en jugadores juveniles de balonmano. Sin embargo, se aprecian valores superiores a los pliegues medidos en gimnastas (Leyton, et al., 2012) y jugadoras de pádel (Pradas, et al., 2014).

Por otro lado, y con el objetivo de encontrar un perfil antropométrico que se relacione con un mayor rendimiento deportivo en waterpolo. Se analizó la relación existente entre diversos parámetros físicos y la consecución de velocidades de lanzamiento superiores. Ya que para Vila, et al. (2009); Piscitelli et al. (2016) y Van der Wende (2005), esta es una de las variables que más influyen en la consecución de un mayor rendimiento deportivo.

En este sentido, la hipótesis de partida indica que en la condición de lanzamiento con oposición (portero), la velocidad de los lanzamientos debería aumentar con el fin de conseguir marcar gol, minimizando el tiempo disponible para bloquear el tiro por parte del portero (Bloomfield, et al., 1990). Hecho que no se aprecia al analizar las diferentes velocidades medidas en los lanzamientos con oposición $(13.63 \mathrm{~m} / \mathrm{s}$ en categoría infantil; $15.23 \mathrm{~m} / \mathrm{s}$ en categoría cadete; $\mathrm{y} 15.17 \mathrm{~m} / \mathrm{s}$ en categoría juvenil), y sin oposición (13.73m/s en categoría infantil; $15.29 \mathrm{~m} / \mathrm{s}$ en cadete; y

Tabla 3.

\begin{tabular}{|c|c|c|c|c|c|c|c|c|c|c|c|c|c|c|c|c|c|c|c|c|c|c|c|}
\hline Variables & 1 & 2 & 3 & 4 & 5 & 6 & 7 & 8 & 9 & 10 & 11 & 12 & 13 & 14 & 15 & 16 & 17 & 18 & 19 & 20 & 21 & 22 & 23 \\
\hline $1 \mathrm{Edad}$ & 1 & $-.58 * *$ & $-.65 * *$ & $-.69 * *$ & -.29 & .35 & $.53^{* *}$ & -.35 & .29 & .29 & -.08 & -.08 & -.08 & $-.75^{* * *}$ & $-.61^{* *}$ & -.26 & $-.80^{* * *}$ & $-.56^{* *}$ & $-.73^{*}$ & $-.80^{* *}$ & $-.75 * *$ & $-.79 * *$ & $-.69 * *$ \\
\hline 2 Categoría & & 1 & $.47 *$ & $.47 *$ & .17 & -.27 & $-.45 *$ & .27 & -.30 & -.30 & .24 & .10 & .10 & $.61^{* *}$ & $.55^{* * *}$ & .14 & $.48^{*}$ & $.40^{*}$ & $.40^{*}$ & .30 & .38 & .35 & .35 \\
\hline 3 masa & & & 1 & $.73^{* *}$ & $.67^{* *}$ & .07 & -.32 & -.07 & .04 & .04 & .37 & -.25 & -.25 & $.97 * *$ & $.77^{* *}$ & $.75^{* *}$ & $.82^{* *}$ & $.61^{* *}$ & $.77 * *$ & $.71^{* *}$ & $.71^{* *}$ & $.71 * *$ & $.71^{* *}$ \\
\hline 4 Talla & & & & 1 & .06 & -.39 & $-.52^{* * *}$ & .39 & $-.43^{*}$ & $-.43^{*}$ & -.19 & $.40^{*}$ & $.40^{*}$ & $.71^{* *}$ & $.72 * *$ & .29 & $.57^{* * *}$ & $.65^{* *}$ & $.96 * *$ & $.56^{* * *}$ & $.68 * *$ & $.58^{* *}$ & $.60^{* *}$ \\
\hline 5 IMC & & & & & 1 & $.474^{*}$ & .01 & $-.47 *$ & $.51^{*}$ & $.51^{*}$ & $.81 * *$ & $-.83^{* *}$ & $-.83 * *$ & $.65 * *$ & .35 & $.86^{* * *}$ & $.64^{* *}$ & .22 & .16 & $.44^{*}$ & .30 & $.40^{*}$ & .37 \\
\hline 6 Pliegues & & & & & & 1 & $.79 * *$ & $-1 * *$ & $.96 * *$ & $.96 * *$ & $.43 *$ & $-.71 * *$ & $-.71^{* * *}$ & .01 & -.09 & $.48^{*}$ & -.04 & -.21 & $-.40^{*}$ & -.15 & -.24 & -.18 & -.29 \\
\hline 7 Jackson & & & & & & & 1 & $-.79 * *$ & $.80^{* * *}$ & $.80 * *$ & -.02 & -.35 & -.35 & -.36 & -.35 & .08 & -.35 & -.31 & $-.52 * *$ & $-.41^{*}$ & $-.45 *$ & $-.43^{*}$ & $-.60^{* *}$ \\
\hline 8 Densidad & & & & & & & & 1 & $-.96 * *$ & $-.96^{* *}$ & $-.43^{*}$ & $.71^{* *}$ & $.71^{* * *}$ & -.01 & .09 & $-.48 *$ & .05 & .21 & $.40^{*}$ & .16 & .23 & .18 & .28 \\
\hline 9 S PC & & & & & & & & & 1 & $1^{* *}$ & $.43^{*}$ & $-.72 * *$ & $-.72 * *$ & .00 & -.10 & $.47 *$ & .02 & -.19 & -.39 & -.10 & -.22 & -.13 & -.28 \\
\hline 10 Endomorfia & & & & & & & & & & 1 & $.43^{*}$ & $-.72 * *$ & $-.72 * *$ & .00 & -.10 & $.46^{*}$ & .02 & -.19 & -.39 & -.10 & -.22 & -.13 & -.28 \\
\hline 11 Mesomorfia & & & & & & & & & & & 1 & $-.85^{* *}$ & $-.85 * *$ & $.41 *$ & .04 & $.53 * *$ & .36 & .03 & -.13 & .13 & .04 & .12 & .15 \\
\hline 12 Ectomorfia & & & & & & & & & & & & 1 & $1^{* *}$ & -.24 & .03 & $-.60^{* *}$ & -.29 & .11 & .30 & -.09 & .02 & -.08 & -.02 \\
\hline 13 CAP & & & & & & & & & & & & & 1 & -.24 & .03 & $-.60^{* *}$ & -.29 & .11 & .30 & -.09 & .02 & -.08 & -.02 \\
\hline 14 MME & & & & & & & & & & & & & & 1 & $.76^{* *}$ & $.69 * *$ & $.87 * *$ & $.61^{* *}$ & $.74 * *$ & $.72 * *$ & $.72 * *$ & $.72 * *$ & $.67 * *$ \\
\hline 15 d_biacromial & & & & & & & & & & & & & & & 1 & $.50^{*}$ & $.71^{* *}$ & $.50^{*}$ & $.75^{* *}$ & $.64 * *$ & $.67 * *$ & $.67 * *$ & $.57 * *$ \\
\hline 16 d_biileocrestal & & & & & & & & & & & & & & & & 1 & $.61 * *$ & $.42^{*}$ & .32 & $.47 *$ & $.41 *$ & $.43 *$ & .32 \\
\hline 17 d_mesoesternal & & & & & & & & & & & & & & & & & 1 & $.56 * *$ & $.66^{* *}$ & $.77 * *$ & $.76^{* * *}$ & $.78^{* *}$ & $.69 * *$ \\
\hline $18 \mathrm{~d}$ dbiestiloideo & & & & & & & & & & & & & & & & & & 1 & $.63 * *$ & $.52 * *$ & $.55 * *$ & $.51 * *$ & $.46^{*}$ \\
\hline 19 envergadura & & & & & & & & & & & & & & & & & & & 1 & $.66^{* *}$ & $.73 * *$ & $.67 * *$ & $.71 * *$ \\
\hline 20 Lanz.SinOp. & & & & & & & & & & & & & & & & & & & & 1 & $.89 * *$ & $.98 * *$ & $.78^{* *}$ \\
\hline 21 Lanz.Op & & & & & & & & & & & & & & & & & & & & & 1 & $.95 * *$ & $.76 * *$ \\
\hline 22 Vel_Lanz. & & & & & & & & & & & & & & & & & & & & & & 1 & $.79 * *$ \\
\hline 23 Dinamometría & & & & & & & & & & & & & & & & & & & & & & & 1 \\
\hline
\end{tabular}

Leyenda: IMC: Índice de masa coporal $\mathrm{kg} / \mathrm{m}^{2}$. Pliegues: valores antropométricos de la medida de los pliegues cutáneos; Jackson: valores de la fórmula de valoración antropométrica propuesta por Jackson y Pollock (1978). Densidad: valores de la fórmula de valoración antropométrica propuesta por Whiter et al. (1987); CAP. valomientos en la condición "sin portero". Lanz.Op.: lanzamientos en la condición "con portero". * $p<.05 ; * * p<.01$. 
$16.50 \mathrm{~m} / \mathrm{s}$ en categoría juvenil). Obteniendo valores $\mathrm{p}>.05$ al comparar las medidas usando el test no paramétrico U de Man Whitney.

Estos resultados son consistentes con los que halla Katrina Van der Wende (2005) y Ferragut et al. (2015), en los que no aprecian diferencias en la velocidad de lanzamiento según la existencia o no de oposición (portero), ni en función de la posición de juego del lanzador ni de lanzamiento (Argudo, García-Cervantes \& Ruíz, 2016). Lo que contrasta con estudios previos (Davis \& Blanksby, 1977), así como con los resultados expuestos por Rivilla et al. (2010), quienes estiman que la localización del lanzamiento en función de la posición del portero, la situación y el grado de oposición, influye en la velocidad de lanzamiento a portería en balonmano.

Al analizar la relación existente entre diferentes pliegues y diámetros antropométricos medidos, se aprecia significación entre el diámetro biacromial y la fuerza de agarre máxima, al igual que se encuentra en el estudio con waterpolistas adultos (Ferragut, et al., 2011). Aunque estos autores también encuentran significación al analizar la fuerza y el diámetro biepicóndilo del fémur, al igual que Vila et al. (2009). Quienes además, hallan correlaciones positivas entre circunferencia del brazo, componente mesomórfico y velocidad de lanzamiento (sin oposición).

En este sentido, en un estudio más reciente desarrollado con los jugadores de los 8 mejores equipos de waterpolo masculino español, Ferragut et al. (2015), hallan correlaciones positivas entre la masa muscular, pliegue de la cresta iliaca y abdominal, el perímetro del brazo relajado, diámetro pectoral y biestiloideo de la muñeca, para la condición de lanzamiento con y sin oposición. En nuestro caso, también observamos que estas dos últimas variables tienen relación con la velocidad de lanzamiento, aunque no se observa en otros estudios previos (Ferragut, et al., 2011; Jürimäe, Hurbo \& Jürimäe, 2009).

En el caso de la situación con oposición (Ferragut, et al., 2015), también hallan correlaciones positivas entre el peso, perímetro del brazo contraído, del antebrazo y de la cintura, así como en la longitud del brazo y la fuerza de agarre. Asimismo en la condición sin oposición se aprecia una correlación positiva entre el diámetro biacromial y la velocidad de lanzamiento.

Por otro lado, para Piscitelli et al. (2016), la masa muscular y las características óseas, influyen en la velocidad de los lanzamientos, no así, la antropometría, masa magra y grasa, al igual que se halla en este estudio. Lo que sugiere que una mejor resistencia de los huesos, especialmente su componente de rigidez, puede ser favorable para el rendimiento. Pues estos parámetros guardan relación con la estabilización de las extremidades inferiores (Davis \& Blanksby, 1977; Elliott \& Armour, 1988), y se asocian a mayores niveles de fuerza.

Este dato contrasta con los obtenidos por Ferragut et al. (2011), lo que puede significar, que estos parámetros pueden tener relevancia en la consecución de velocidades de lanzamiento mayores en categorías de formación, pero no en categorías absolutas. Por este motivo es necesario ampliar la muestra en futuros estudios, así como analizar la velocidad de lanzamiento durante la competición. Ya que estos datos nos sugieren que las diferencias en el rendimiento en este deporte (en etapas de formación) hay que buscarlas en relación a otros factores, como la condición física, el dominio técnico y/o táctico, tal y como concluye Bloomfield et al. (1990).

Del mismo modo, destaca la no aparición de diferencias significativas al considerar la variable categoría, lo que demuestra que las medidas de cada subgrupo son homogéneas. Y que el aumento de velocidad hallado a medida que los deportistas son mayores se debe a un crecimiento musculary óseo que se transforma en una aumento de la fuerza. Tal y como expone Gómez et al. (2011), quien aprecia una relación entre el pico de fuerza alcanzado y la velocidad del lanzamiento al normalizar estas variables respecto al peso y/o la altura de los sujetos.

En este sentido, se aprecian similares resultados a los reportados por Bloomfield et al. (1990), quienes en una muestra de jugadores australianos sub19 no encuentran significación entre la suma de los pliegues y la velocidad de lanzamiento, así como en las variables somatotípicas medidas y calculadas. Los resultados reportados por estos autores, hallan elevadas correlaciones entre los factores antropométricos y la velocidad de lanzamiento. Pero solo aprecian significación entre los tres diámetros considerados en la situación de lanzamientos con oposición.

Las principales diferencias, entre categorías se han hallado en los perímetros y pliegues cutáneos. Resultados que reflejan que la principal diferencia, en relación al aumento de velocidad de los lanzamientos, parece estar en los niveles de fuerza, y no en los factores antropométricos. Los valores en los diámetros y perímetros de este estudio (tabla 1) son inferiores a los encontrados por Lozovina y Pavièiæ (2004), en waterpolistas croatas, así como en los jugadores de la selección española absoluta medidos por Ferragut et al. (2011).

En relación a la media de la velocidad de los lanzamientos observados (13.68m/s en categoría infantil; $15.23 \mathrm{~m} / \mathrm{s}$ en categoría infantil; y $16.91 \mathrm{~m} / \mathrm{s}$ en categoría juvenil) se aprecian velocidades inferiores a las reportadas en el estudio realizado por Ferragut et al. (2011), para el combinado nacional absoluto ( $20.53 \mathrm{~m} / \mathrm{s}$ ), así como las reportadas por Whiting, Puffer, Finerman, Gregor y Maletis (1985), para el equipo absoluto norteamericano $(19.7 \mathrm{~m} / \mathrm{s})$. Si bien se ha de comentar que hablamos de deportistas en formación y en edad de crecimiento.

Por otro lado, al comparar la existencia de relación entre la velocidad de lanzamiento y fuerza máxima de agarre se aprecia significación entre ambas medidas y condiciones ( $\mathrm{p}=.82 ; \mathrm{p}<.01$ ), para la condición sin portero; $\mathrm{y}(\mathrm{p}=.80 ; \mathrm{p}<.01)$, si atendemos a la condición con oposición. Lo que demuestra nuevamente que la fuerza es un factor limitante en la velocidad del lanzamiento.

Al contrario de lo reportado por Ferragut et al. (2011), se aprecian correlaciones significativas en las dos condiciones consideradas, lanzamiento con oposición ( $p=.76$; $p<.01)$; $y$ sin oposición $(p=.78 ; p<.01$ ), respecto a la fuerza de agarre máxima. Aunque no se ha podido encontrar otra situación táctica similar, por lo que entendemos que el tamaño del balón y distancia a portería puede condicionar de manera importante la velocidad alcanzada en los disparos siendo necesaria mayor investigación al respecto.

Por otro lado, y salvo el estudio de Ferragut et al. (2011), cuya muestra son jugadores de élite de mayor edad, no se han encontrado estudios similares para este deporte. Aunque es de reseñar la relación que encuentra (Pugh, Kovaleski, Heitman \& Pearsall 2001) entre velocidad de lanzamiento y fuerza máxima de agarre para pitchers femeninas de béisbol, así como Mikkelsen y Olesen (1976) para jugadoras de balonmano.

Por todo lo expuesto anteriormente se pude concluir que los factores antropométricos no modificables por el entrenamiento (diámetros óseos) son necesarios para llegar a la élite en este deporte. Así cómo es posible apreciar diferencias en cuanto al nivel de los deportistas en función de la velocidad de lanzamiento y la fuerza máxima de agarre desarrollada. Aun así, no es posible determinar el éxito deportivo futuro en función de las características somatotípicas en estas edades al contrario que para jugadores de élite absolutos.

\section{Limitaciones}

Una de las limitaciones con la que cuenta este estudio radica en que no se ha tenido en cuenta la edad madurativa de los participantes. Por lo que la interpretación de los resultados debe tomarse con cautela, ya que la pubertad puede alterar el desarrollo de la fuerza de los participantes. Estos aspectos, que suponen en parte algunas de las limitaciones del estudio, no menoscaban la potencialidad de los resultados y la posible influencia de los parámetros físicos modificables en el entrenamiento (altura, envergadura, masa corporal, pliegues cutáneos y perímetros corporales) en la consecución de una mayor velocidad de lanzamiento y mayor fuerza máxima de agarre, las cuales determinarán el rendimiento futuro del deportista.

En este sentido cobra especial relevancia para los entrenadores y seleccionadores de deportistas en edades de formación conocer el perfil antropométrico preponderante para la consecución de un mayor rendimiento deportivo. Así como podría ser interesante trasladar esta información al diseño de rutinas de entrenamiento más eficaces, ya que se ha 
observado que los deportistas usan diferentes patrones de movimiento y lanzamiento para obtener la velocidad máxima de disparo en función de la localización del mismo (Ferragut, et al., 2015, Argudo, et al., 2016).

Futuras líneas de investigación deberían considerar el análisis del tipo de lanzamiento efectuado mediante el visionado a cámara lenta de cada secuencia de lanzamiento desde un plano lateral (Rivilla, et al., 2010). Teniendo en cuenta las dos condiciones consideradas y no sólo la velocidad de los lanzamientos, sino su eficacia anotadora tal y como sugieren Piscitelli et al. (2016). Ya que Van der Wende (2005), aprecia diferencias cualitativas en los lanzamientos, en función de la existencia o no de oposición.

No obstante, en este estudio, no es posible encontrar diferencias de carácter significativo entre los lanzamientos realizados en las distintas posiciones seleccionadas (lado derecho, centro y lado izquierdo de la portería), al igual que halla Argudo et al. (2016). Aunque si es de remarcar, que se aprecian diferencias entre la condición de lanzamiento sin oposición y con oposición. Apreciándose un índice de correlación rho significativo, entre ambos factores $(\mathrm{p}=.885 ; \mathrm{p}<.001)$.

Del mismo modo debería considerarse el factor fatiga dentro del estudio de la eficiencia y precisión anotadora. García, Moreno, Reina y Menayo (2011), aprecian que en una tarea de lanzamiento en cricket, los sujetos alcanzaron su mayor precisión lanzando entre el 75-85\% de su velocidad máxima, constatando igualmente que un aumento en la velocidad del lanzamiento no implicaba una disminución en la precisión del mismo. Asimismo, la eficacia en la localización del lanzamiento estaba afectada por la práctica, disminuyendo la precisión gradualmente. Sin embargo, la velocidad de lanzamiento se mantuvo estable a lo largo del tiempo (García, et al., 2011; Gómez, et al., 2011).

\section{Referencias}

Abraldes, J.A., Rodríguez, N., Ferragut, C., \& Vila, H. (2014). Características antropométricas, composición corporal y somatotipo en deportistas de élite de salvamento. Retos: nuevas tendencias en educación física, deporte y recreación,(26), 66-70.

Argudo, F. M. (2002). Análisis del rendimiento en competición en deportes colectivos. Su aplicación al waterpolo. Comunicaciones técnicas, 3, 83-90.

Argudo, F. M., \& García, P. (2013). Propuestas de reglamento para categorías prebenjamín, benjamín, alevín e infantil en waterpolo. Comunicaciones Técnicas, 3, 13-20.

Argudo, F. M., García-Cervantes, L., \& Ruíz, E. (2016). Factores asociados a la eficacia de gol en waterpolo. Retos: nuevas tendencias en educación física, deporte y recreación, (29), 105-108

Argudo, F. M., Ruiz, E., \& Abraldes, J. A. (2010). Influencia de los valores de eficacia sobre la condición de ganador o perdedor en un mundial de Waterpolo. Retos. Nuevas tendencias en Educación Física, Deporte y Recreación, 17, 21-24.

Bloomfield, J., Blanksby, B. A., Ackland, T. R., \& Allison, G T. (1990). The influence of strength training on overhead throwing velocity of elite water polo players. Australian Journal of Science and Medicine in Sport, 22(3), pp. 63-67.

Carter, J., \& Heath, B. (1990). Somatotyping: development and applications (Vol. 5). Cambridge University Press.

Chacón-Araya, Y., Fernández-Campos, C., \& Moncada-Jiménez, J. (2016). Características cineantropométricas e ingesta nutricional de mujeres y hombres costarricenses dedicados al modelaje publicitario. Retos. Nuevas tendencias en Educación Física, Deporte y Recreación, (30), 144-149.

Davis, T., \& Blanksby, B. A. (1977). A cinematographic analysis of the overhand water polo throw. Journal of Sports Medicine and Physical Fitness, 17(1), 5-16

Domínguez, J.A., Sánchez, L., Rodríguez, D., \& González, J. J. (2015). Variables antropométricas y de rendimiento físico en niños y niñas de 10-15 años de edad. Retos: nuevas tendencias en educación física, deporte y recreación, (27), 86-92.

Elliott, B., \& Armour, J. (1988). The penalty throw in water polo: a cinematographic analysis. Journal of Sports Sciences, 6(2), 103114.

Feltner, M. E., \& Taylor, G (1997). Three-dimensional kinetics of the shoulder, elbow, and wrist during a penalty throw in water polo. Journal of Applied Biomechanics, 13(3), 347-372.

Ferragut, C., Abraldes, J. A., Manchado, C., \& Vila, H. (2015). Water polo throwing speed and body composition: an analysis by playing positions and opposition level. Journal of Human Sport and Exercise, 10(1), 81-94.

Ferragut, C., Vila, H., Abraldes, J. A., Argudo, F., Rodriguez, N., \& Alcaraz, P. E. (2011). Relationship among maximal grip, throwing velocity and anthropometric parameters in elite water polo players. Journal of Sports Medicine and Physical Fitness, 51(1), 26-32.

García, P. (2009). Evaluación cuantitativa de la desigualdad numérica temporal simple con posesión mediante observación sistemática en waterpolo. (Tesis doctoral, Universidad Autónoma de Madrid, Madrid, España).

García, M., Alcaraz, P. E., Ferragut, C., Manchado, C., Abraldes, J. A., Rodríguez, N., \& Vila, H. (2011). Composición corporal y velocidad de lanzamiento en jugadoras de élite de balonmano. Cultura_Ciencia_Deporte, 6(17), 129-135.

García, J. A., Moreno, F. J., Reina, R., \& Menayo, R. (2011). La velocidad y la precisión en el lanzamiento en jóvenes jugadores de balonmano en función de la concentración de la práctica. Retos: nuevas tendencias en educación física, deporte y recreación, (19), 43-46.

Gómez, J. S., Sabido, R., Gómez-Valadés, J. M., \& Barbado, D. (2011). Influencia aguda de la aplicación de un tratamiento de fuerza basado en el método de contrastes combinado, sobre la precisión y la velocidad del lanzamiento en balonmano. Revista de Ciencias del Deporte, 7(1), 5-16.

Gorostiaga, E. M., Granados, C., Ibanez, J, Gonzalez-Badillo, J. J., \& Izquierdo, M. (2006). Effects of an entire season on physical fitness changes in elite male handball players. Medicine and Science in Sports and Exercise, 38(2), 357.

Gorostiaga, E. M., Granados, C., Izquierdo, M., Ibañez, J., \& Bonnabau, H. (2007). Differences in physical fitness and throwing velocity among elite and amateur female handball players. International Journal of Sports Medicine, 28(10), 860-867.

Hohmann, A., \& Frase, R. (1992). Analysis of swimming speed and energy metabolism in competition water polo games. Swimming science VI: biomechanics and medicine in swimming. E\& FNSpon, London, 313-319.

Jackson, A. S., \& Pollock, M. L. (1978). Generalized equations for predicting body density of men. British journal of nutrition, 40(3), 497-504.

Joris H. J., van Muijen, A. J., van Ingen Schenau G. J., \& Kemper H. C. (1985). Force, velocity and energy flow during the overarm throw in female hand ball players. Journal of Biomechanics, 18, 409-14.

Jürimäe, T., Hurbo, T., \& Jürimäe, J. (2009). Relationship of handgrip strength with anthropometric and body composition variables in prepubertal children. HOMO-Journal of Comparative Human Biology, 60(3), 225-238.

Lee, R. C., Wang, Z., Heo, M., Ross, R., Janssen, I., \& Heymsfield, S. B. (2000). Total-body skeletal muscle mass: development and cross-validation of anthropometric prediction models. The American Journal of Clinical Nutrition, 72(3), 796-803.

Leyton, M., Luis, V., Sabido, R., \& Morenas, J. (2012). Perfil y diferencias antropométricas y físicas de gimnastas de tecnificación de las modalidades de artística y rítmica. Retos: nuevas tendencias en educación física, deporte y recreación, (21), 58-62.

Lloret, M. (1994). Análisis de la acción de juego en el waterpolo durante la Olimpiada de 1992. Universidad de Barcelona, Tesis Doctoral.

Lloret, M. (1995). Análisis praxiológico del waterpolo. Revista de Entrenamiento Deportivo 8, 28-36.

Lozovina, M., \& Pavièiæ, L. (2004). Antropometric changes in Elite 
male water polo players: Survey in 1980 and 1995. Croatian Medical Journal, 45(2), 202-205.

Lozovina, M., Lozovina, V., \& Pavièiæ, L. (2012). Morphological changes in elite male water polo players: Survey in 1980 and 2008.

Martin, A. D., Spenst, L. F., Drinkwater, D. T., \& Clarys, J. P. (1990). Anthropometric estimation of muscle mass in men. Medicine and Science in Sports and Exercise, 22(5), 729-733.

Mikkelsen, F., \& Olesen M. N. (1976). Handball 82-84 (Traening af skudstyrken). Stockholm: Trygg-Hansa.

Montero, I., \& León, O. (2007). A guide for naming research studies in psychology. International Journal of Clinical Healthy Psychology, 7(3), 847-862.

Norton K., \& Olds T. (2001). Morphological evolution of athletes over the $20^{\text {th }}$ century: causes and consequences. Sports Medicine, 31, 763-83.

Padilla, J. R. (2010). Perfil de proporcionalidad y la velocidad del lanzamiento en jugadores de béisbol. Revista Internacional de Medicina y Ciencias de la Actividad Física y el Deporte 10(37), 93-116.

Piscitelli, F., Milanese, C., Sandri, M., Cavedon, V., \& Zancanaro, C. (2016). Investigating predictors of ball-throwing velocity in team handball: the role of sex, anthropometry, and body composition. Sport Sciences for Health, 12(1), 11-20.

Pradas, F., Cachón, J., Ortín, D., Quintas, A., Arraco, S. I., \& Castellar, C. (2014). Análisis antropométrico, fisiológico y temporal en jugadoras de pádel de elite. Retos: nuevas tendencias en educación física, deporte y recreación, (25), 107-112.

Pugh S. F., Kovaleski, J. E., Heitman, R.J., \& Pearsall A.W. (2001). Upper and lower body strength in reltation to underban pitching speed by experienced and inexperienced pitcher. Perceptual and motor Skill, 93, 813-8.

Rivilla, J., Sampedro, J., Navarro, F., \& Gómez, M. J. (2010). Influencia de la oposición en la velocidad de lanzamiento en jugadores de balonmano de élite, amateur y formación. Revista Internacional de Ciencias del Deporte. 18(6), 91-99. Acta Kinesiologica, 6(2), 85-90.

Salkind, N. (1999). Métodos de investigación. México: Prentice Hall. Smith H. K. (1998). Applied physiology of water polo. Sports Medicine, 26, 317-34.

Tsekouras, Y. E., Kavouras, S. A., Campagna, A., Kotsis, Y. P., Syntosi, S. S., Papazoglou, K., \& Sidossis, L. S. (2005). The anthropometrical and physiological characteristics of elite water polo players. European Journal of Applied Physiology, 95(1), 35-41.

Van der Wende, K. (2005). The effects of game-specific task constraints on the outcome of the water polo shot. Auckland University of Technology, New Zeland.

Van Muijen, A. E., Joris, H., Kemper, H. C., \& Van Ingen Schenau, G J. (1991). Throwing practice with different ball weights: Effects on throwing velocity and muscle strength in female handball players. Sports Medicine, Training and Rehabilitation, 2(2), 103-113.

Vila, H., Abraldes, J. A., \& Rodríguez, N. (2009). Estudio del perfil antropométrico del jugador juvenil de balonmano en la Región de Murcia. Retos: nuevas tendencias en educación física, deporte y recreación, (16), 80-85

Vila, H., Ferragut, C., Abraldes, J. A., Rodriguez, N., \& Argudo, F. M. (2010). Anthropometric characteristics of elite players in water polo. International Journal of Medicine and Science of Physical Activity and Sport, 10(40), 652-663.

Vila, H., Ferragut, C., Argudo, F. M., Abraldes, J. A., Rodríguez, N., \& Alacid, F. (2009). Relación entre parámetros antropométricos y la velocidad de lanzamiento en jugadores de waterpolo. Journal of Human Sport and Exercise, 4(1), 62-74.

Wallace, M., \& Cardinale, M. (1997). Conditioning for Team Handball. Strength \& Conditioning Journal, 19(6), 7-12.

Whiting, W. C., Puffer, J. C., Finerman, G, Gregor, R. J., \& Maletis, G. B. (1985). Three-dimensional cinematographic analysis of water polo throwing in elite performers. The American Journal of Sports Medicine, 13(2), 95-98.

Withers, R. T., Craig, N. P., Bourdon, P. C., \& Norton, K. I. (1987) Relative body fat and anthropometric prediction of body density of male athletes. European Journal of Applied Physiology, 56, 191200.
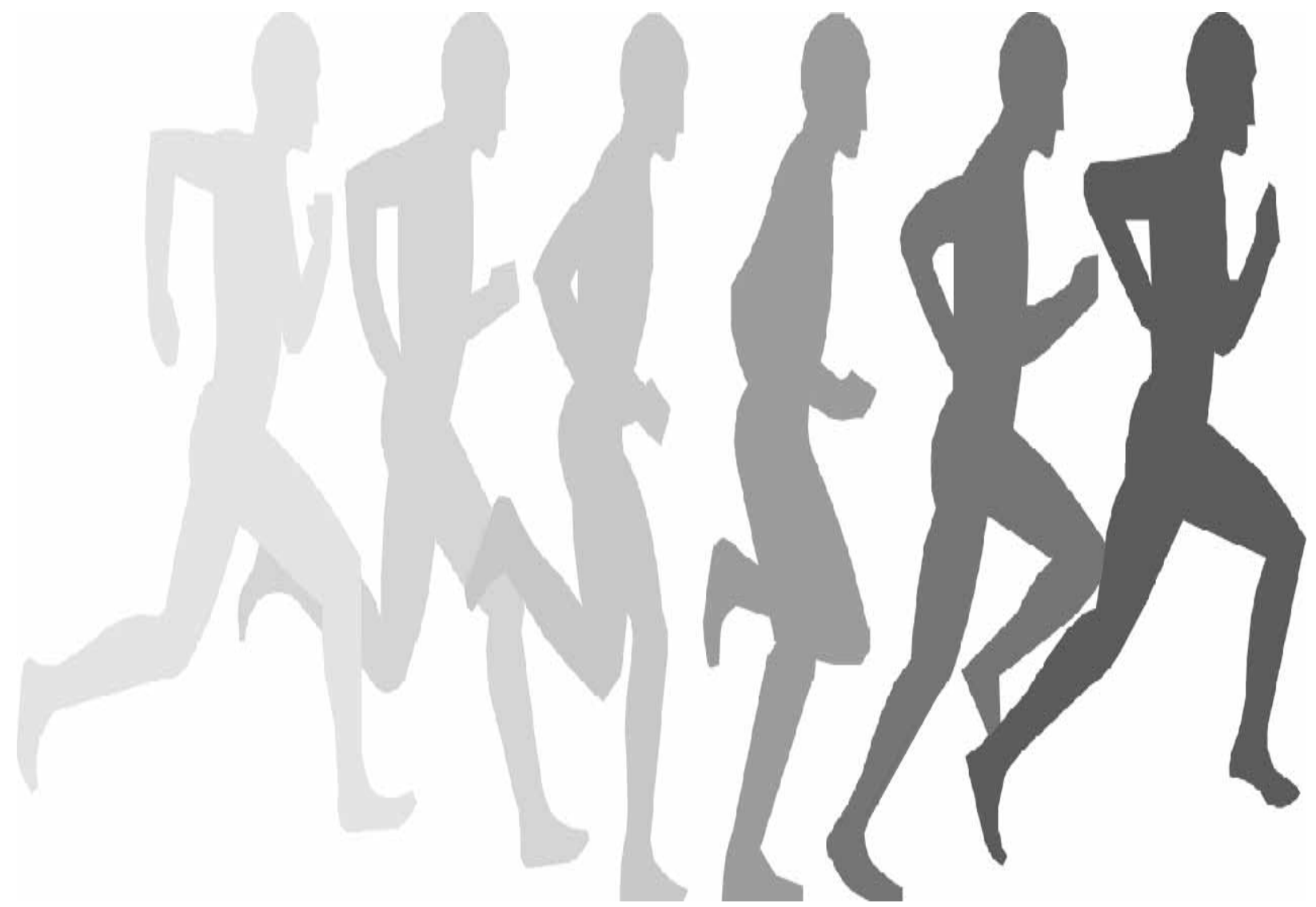